\title{
Un quinquenio de experiencia (2005-2010) con infecciones por Salmonella spp en un centro nacional de referencia en pediatría
}

\author{
Patricia Barrios, Federica Badía, Valentina Misa, M. Inés Mota, Arací Martínez, \\ Hebert Mariño, Gabriela Algorta, Javier Prego y M. Catalina Pírez
}

\section{A five-year experience with zoonotic Salmonella at a pediatric reference centre}

Background: Salmonella can cause asymptomatic infections, diarrhea, bacteremia and focal infections such as meningitis and osteomyelitis. Aim: To describe clinical and microbiological aspects of infections by Salmonella spp. in children in a pediatric referral hospital: Centro Hospitalario Pereira Rossell, in Montevideo, Uruguay. Materials and Methods: Descriptive and retrospective study of 46 patients, from which Salmonella spp was isolated between January 1, 2005 and December 31, 2010. Results: Salmonella spp was isolated in 46 children younger than 15 years old. 18 were below 2 years old and 5 children below three months. $24 \%$ of the children had risk factors, such as HIV infection, oncological diseases and malnutrition; low birth weight and pneumonia were associated conditions. No deaths were reported. The serotypes more frequently found were: Typhimurium and Enteritidis. Most of the strains were susceptible to ampicillin and third generation of cephalosporins. Discussion: Diarrhea with blood was the predominant clinical presentation, and there were no outbreaks. Typhimurium and Enteritidis were the most common serotypes. Based on the profiles of susceptibility antimicrobial, we could maintain the same recommendations until the moment suggested. Conclusions: we must consider the Salmonella infection in febrile children under risk of an invasive bacterial disease, with or without focal infection.

Key words: Salmonella, isolates, children.

Palabras clave: Salmonella, aislamientos, niños.

\section{Introducción}

$S$ almonella no tifoidea puede causar infecciones asintomáticas, gastroenteritis, bacteriemia e infecciones focales como meningitis y osteomielitis ${ }^{1-4}$. La enfermedad invasora es más frecuente bajo tres meses de edad, en los portadores de enfermedades crónicas y en los inmunodeprimidos ${ }^{1,5-8}$.

La mayoría de los casos son esporádicos ${ }^{6}$, pero se han comunicado brotes hospitalarios o institucionales. Esto varía según el país y están vinculados a la disponibilidad de agua potable, la conservación de los alimentos, la higiene, el clima, y el suministro de antimicrobianos a animales y humanos, que alteran la microbiota normal.

El diagnóstico microbiológico se realiza por aislamiento del microorganismo en coprocultivo, hemocultivo, urocultivo o muestras de otros focos de infección 9 .

El tratamiento antimicrobiano está indicado en las poblaciones de riesgo de infección diseminada ${ }^{1,6,7}$.

En Uruguay, desde las primeras décadas del siglo XX, la investigación de Salmonella spp se incluyó en estudios etiológicos de niños con diarrea. Estas investigaciones contribuyeron a determinar la frecuencia y la gravedad de la infección por Salmonella enterica en los lactantes (Doctrina
Montevideo) y se realizaron valiosos aportes al conocimiento y descripción de estos agentes y sus variedades (Salmonella Cerro, Salmonella Montevideo, Salmonella Carrau, Salmonella Berta, etc.) Los estudios nacionales publicados hasta principios de 1950 muestran la presencia de Salmonella spp y Shigella spp, únicos agentes investigados. A partir de 1970 la frecuencia de Salmonella en las diferentes publicaciones mostró una drástica reducción porcentual con la incorporación de la investigación de otros potenciales patógenos entéricos y las medidas para controlar la diseminación de los microorganismos en las salas de internación pediátrica. Salmonella sp puede considerarse hoy una causa poco común de gastroenteritis en niños, pero sigue siendo un agente a tener presente en gastroenteritis y en enfermedades transmitidas por alimentos ${ }^{5}$.

\section{Objetivo}

Describir los aspectos microbiológicos y presentación clínica de las infecciones por Salmonella spp en niños internados en el hospital de referencia pediátrico del subsector público de Uruguay (Hospital Pediátrico-Centro Hospitalario Pereira Rossell-HP-CHPR) en un período de cinco años desde el 1 de enero de 2005 al 31 de diciembre de 2010 .
Universidad de la República, Montevideo, Uruguay. Departamento de Pediatría (PB, $F B, V M, H M, M C P)$.

Departamento de Bacteriología y Virología (MIM, AM, GA, JP). Centro Hospitalario Pereira Rossell, Montevideo, Uruguay (PB, FB, VM, HM, MCP).

Laboratorio Microbiología (MIM, AM, GA).

Departamento de Emergencia (JP).

Financiamiento: No hubo Los autores declaran no tener conflictos de interés en este artículo.

Recibido: 20 de marzo de 2017 Aceptado: 27 de junio de 2017

Correspondencia a: Patricia Barrios Godoy patriciabarrios77@gmail.com 


\section{Materiales y Métodos}

Se realizó un estudio descriptivo, retrospectivo. Se analizaron las historias clínicas de 46 pacientes en los que se aisló Salmonella spp de todas las muestras recibidas en el Laboratorio de Microbiología (heces, sangre, otras) entre el 1/1/2005 y el 31/12/2010.

Los registros se obtuvieron del Laboratorio de Bacteriología del HP- CHPR, de las historias clínicas, y de los registros de egresos informatizados (Pediasis) del HPCHPR. Se analizaron las siguientes variables: edad, sexo, procedencia, época del año, asistencia ambulatoria o admisión hospitalaria, días de internación, co-morbilidades, síntomas y signos, tratamiento antimicrobiano, datos de laboratorio incluyendo serovariedad, y susceptibilidad in vitro.

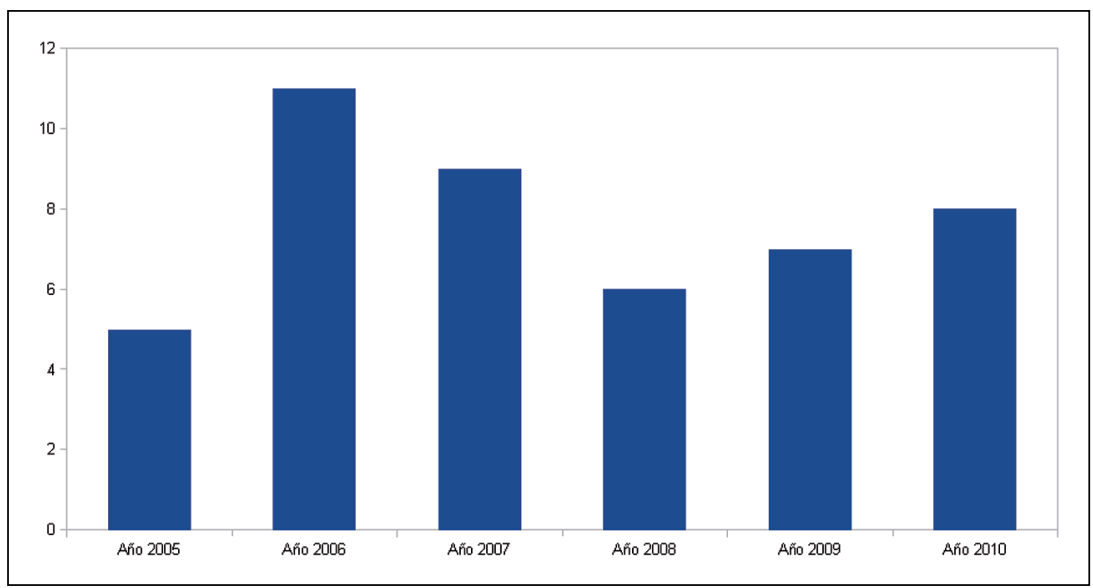

Figura 1. Aislamientos de Salmonella spp en menores de 15 años de edad. Distribución anual. Hospital Pediátrico Centro Hospitalario Pereira Rossell, 2005-2010. Uruguay.

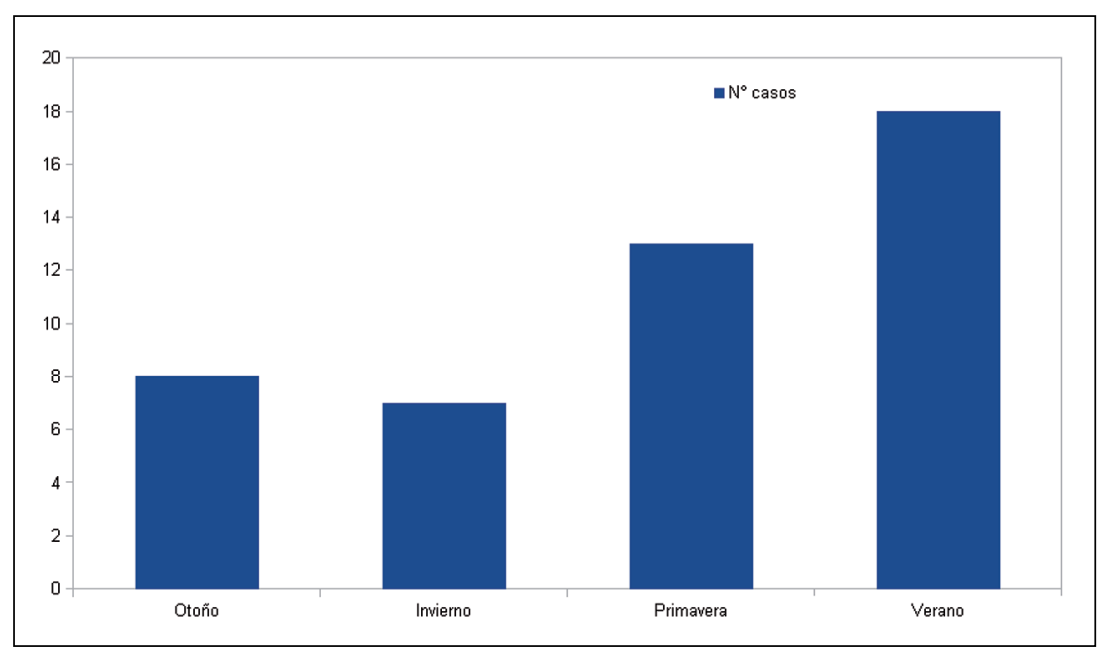

Figura 2. Aislamientos de Salmonella spp en menores de 15 años de edad. Distribución según estaciones del año. Hospital Pediátrico Centro Hospitalario Pereira Rossell, 2005-2010. Uruguay.
Para la definición de las diferentes entidades clínicas: gastroenteritis, sepsis, shock séptico, bronquiolitis, neumonía, osteomielitis, fiebre sin foco evidente se siguieron las recomendaciones publicadas en las normas nacionales de atención pediátrica ${ }^{10-15}$. Se definió bacteriemia oculta, como la presencia de una bacteria en sangre sin signos de toxicidad y sin evidencia de foco infeccioso ${ }^{16}$.

Los datos se procesaron en planilla Excel y se realizaron cálculos de frecuencias y proporciones de variables continuas y discontinuas.

\section{Resultados}

Se aisló Salmonella enterica en 46 niños bajo 15 años de edad. De éstos, 28 aislados provenían de heces, 15 en hemocultivos, dos de punciones osteo-articulares y uno de un absceso de un miembro inferior.

Se pudieron analizar las características microbiológicas de los 46 casos, pero en ocho pacientes que fueron asistidos en forma ambulatoria, no se pudo obtener toda la información clínica.

De los 46 niños, 52\% (n: 24) eran varones. La media de edad fue de 37 meses, con un rango de 1 mes a 14 años. El 39\% (n: 18) eran pacientes con edad inferior a dos años y cinco niños tenían menos de tres meses.

Se registraron entre 5 y 11 casos por año; ninguno de los casos fue asociado a un brote (Figura 1). En cuanto a la distribución estacional, $39 \%$ de los casos ocurrieron en verano, $28 \%$ en primavera, $17 \%$ en otoño y $15 \%$ en invierno (Figura 2). El $84 \%$ de los niños procedía de Montevideo (Figura 3).

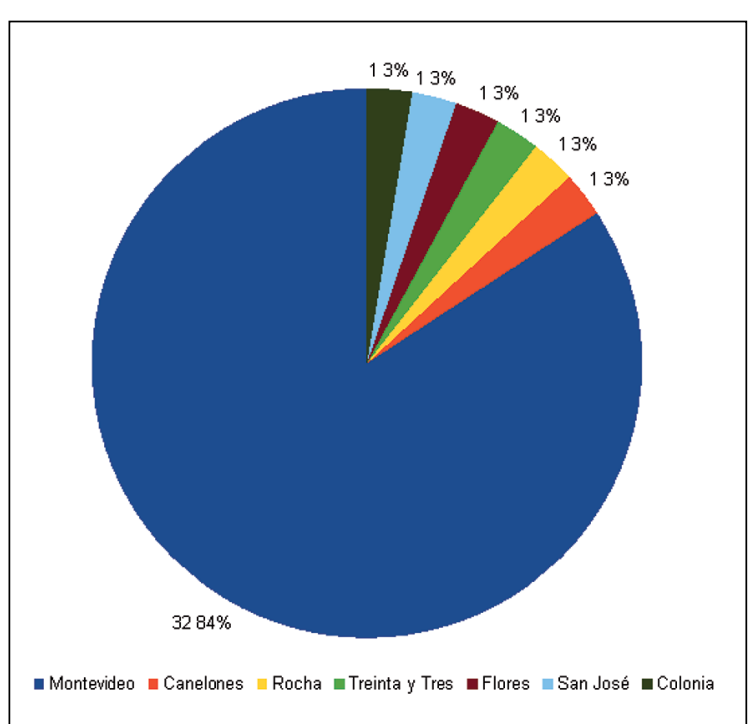

Figura 3. Aislamientos de Salmonella spp en menores de 15 años de edad. Distribución según departamento de residencia. Hospital Pediátrico Centro Hospitalario Pereira Rossell, 2005-2010 Uruguay. 


\begin{tabular}{|c|c|c|}
\hline Síntomas y signos clínicos & Total & $\%$ \\
\hline Fiebre & 16 & 42 \\
\hline Deposiciones con sangre & 19 & 50 \\
\hline Deposiciones sin sangre & 7 & 18 \\
\hline Vómitos & 11 & 29 \\
\hline Dolor abdominal & 3 & 7 \\
\hline Deshidratación & 9 & 24 \\
\hline
\end{tabular}

De los 38 pacientes en que se pudo obtener todos los registros de la historia clínica, 26 (68\%) consultaron por gastroenteritis aguda (GEA), y la mayoría de éstos (73\%) tenía sangre en las deposiciones.

Casi la mitad (42\%) presentaba fiebre mayor de 38 ${ }^{\circ} \mathrm{C}$. Los síntomas y signos más frecuentes se describen en la Tabla 1.

Fueron hospitalizados 26 de los 38 pacientes (68\%). Los motivos de ingreso fueron: GEA con fiebre 17 (65\%), de los cuales 10 presentaban sangre en las deposiciones, cinco por fiebre sin foco, dos pacientes presentaban osteomielitis (fémur y tobillo), un paciente con diagnóstico de bronquiolitis había cursado diarrea la semana previa, y un paciente tenía neumonía. La media de edad de estos 26 niños hospitalizados fue de 39 meses.

Dos de estos pacientes requirieron internación en unidad de cuidado intensivo ya que evolucionaron con un shock séptico: un recién nacido de un mes de vida y un lactante de tres meses.

La internación tuvo una duración media de 11 días, con un rango de 2 a 43 días.

Respecto a las co-morbilidades vinculadas a riesgo de infección invasora por Salmonella sp, se describieron: dos pacientes con infección por VIH; una niña de 1 año y 9 meses, sin inmunosupresión y otro de 2 años con inmunosupresión en tratamiento anti-retroviral (TARV); tres pacientes presentaban una enfermedad hematooncológica: dos con leucemia linfoblástica aguda y uno un neuroblastoma.

Tres niños presentaban desnutrición y un paciente tenía antecedente de bajo peso al nacer, asma del lactante y neumonía.

En la Tabla 2 se observa las características clínicas y microbiológicas de los pacientes con bacteriemia por Salmonella sp.

Cuatro niños (36\%) tuvieron bacteriemia oculta, definida como la presencia de una bacteria en sangre sin signos de toxicidad.

De los 26 pacientes hospitalizados, 20 (77\%) recibie-

\begin{tabular}{|c|c|c|c|c|}
\hline & Edad & Diagnóstico & Co-morbilidad & Serotipo \\
\hline 1 & $5 a$ & Fiebre sin foco & LLA $^{a}$ & Enteritidis \\
\hline 2 & $7 \mathrm{~m}$ & Neumonía & $\mathrm{BPN}^{b}, \mathrm{CBO}^{c}$, neumonía & Oranienburg \\
\hline 3 & $1 \mathrm{~m}$ & GEAd shock séptico & DNT & Sin tipificar \\
\hline 4 & $5 a$ & Fiebre $\sin$ foco & Neuroblastoma & Typhimurium \\
\hline 5 & $2 a$ & Celulitis & & Typhimurium \\
\hline 6 & $3 \mathrm{~m}$ & Shock séptico + rotavirus & & Typhimurium \\
\hline 7 & $2 a$ & GEA repercusión general & VIH-TARV ${ }^{f}$ & Enteritidis \\
\hline 8 & $8 \mathrm{~m}$ & $\mathrm{BQ}^{9} \mathrm{VRS}+$ repercusión general & & Enteritidis \\
\hline 9 & $10 \mathrm{~m}$ & Osteoartritis & & Enteritidis \\
\hline 10 & $3 \mathrm{~m}$ & Fiebre $\sin$ foco & & Agona \\
\hline 11 & $8 a$ & Neutropenia febril & LLA & Typhimurium \\
\hline
\end{tabular}

ron tratamiento con antimicrobianos, $50 \%$ con ceftriaxona i/v, el resto recibió azitromicina, cefuroxima, ampicilina, amoxicilina, y dos combinaciones de cefalosporina de tercera generación + aminoglucósido.

Los serotipos más frecuentemente aislados fueron: Typhimurium 26 (56\%) y Enteritidis 14 (30\%). Los demás serotipos aislados fueron: Oranienburg, Newport, Montevideo, Muenchen, Bredeney y Agona.

La mayoría de las cepas eran sensibles a ampicilina, cefalosporinas de tercera generación y cotrimoxazol. En los años 2005, 2006 y 2009 no se aislaron cepas resistentes. En las Figuras 4 y 5 se observan la distribución por año de los serotipos y su susceptibilidad antimicrobiana in vitro.

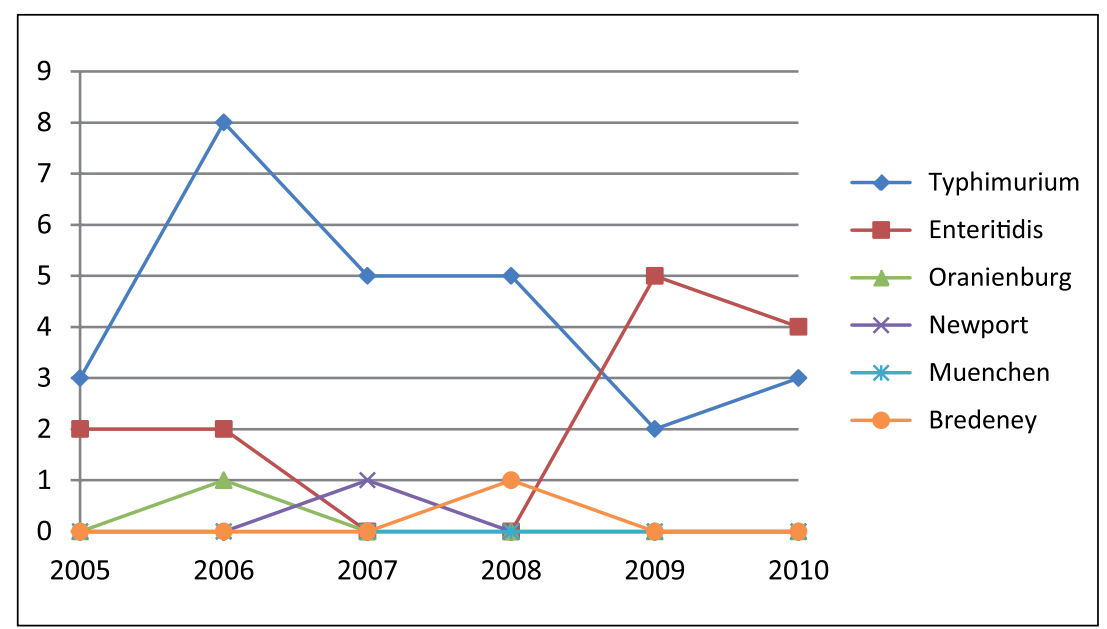

Figura 4. Distribución anual de los serotipos de Salmonell spp aislados en laboratorio de microbiología del CHPR entre los años 2005-2010. 


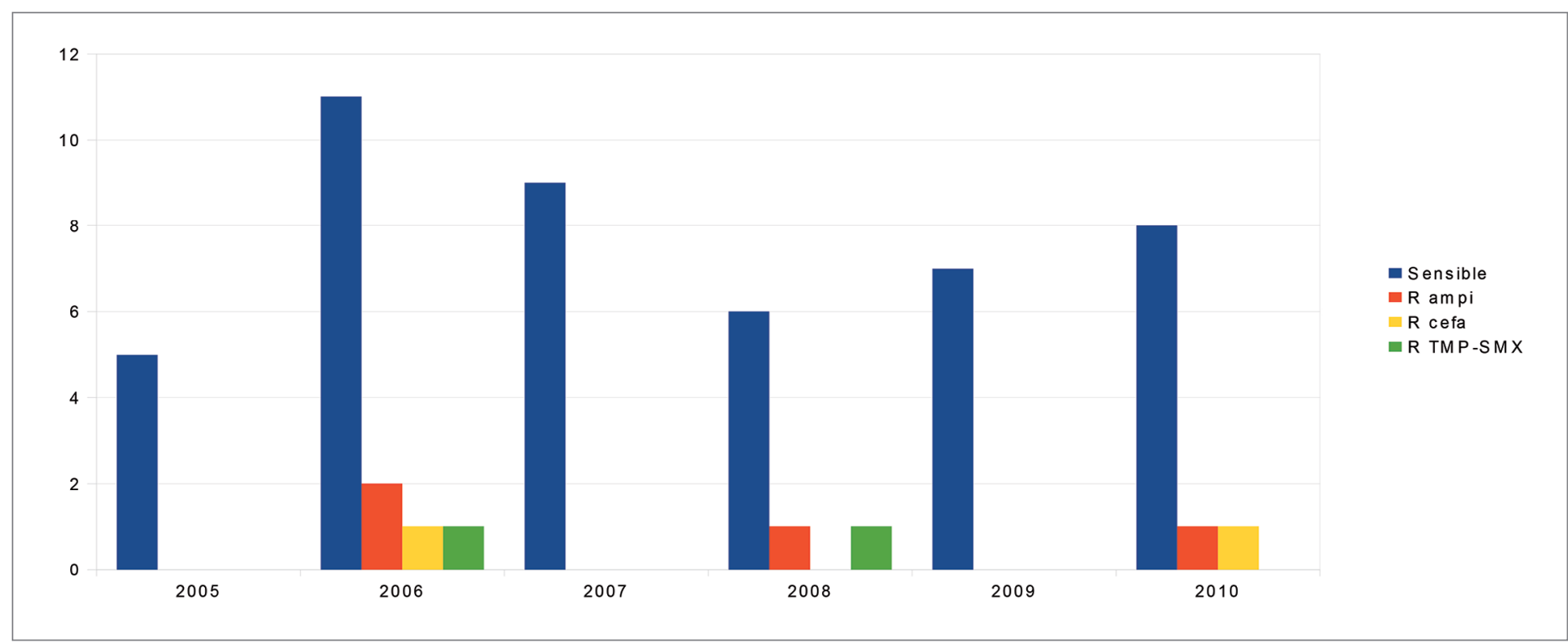

Figura 5. Susceptibilidad antimicrobiana de los aislados de Salmonella spp del Laboratorio de Microbiología del CHPR entre los años 2005 y 2010.

\section{Discusión}

Se estima que la incidencia global de Salmonella sp zoonótica es de 93,8 millones de casos de gastroenteritis con una mortalidad de 155.000 muertes por año ${ }^{1,6}$.

Existe un aumento de la incidencia de salmonelosis que estaría vinculado a la producción de alimentos a gran escala, el uso indiscriminado de antimicrobianos, y el aumento de los individuos inmunocomprometidos en la población ${ }^{1}$.

Si bien se describen múltiples formas de adquirir esta infección, la contaminación de los alimentos sigue siendo la causa más importante ${ }^{1,11}$.

En nuestro país, Salmonella enterica es la segunda causa más frecuente de diarrea con sangre luego de Shigella spp. ${ }^{12}$.

En nuestra serie de casos predominaron los varones bajo 2 años de edad, inmunocompetentes. La presentación clínica predominante fue la diarrea con sangre, siendo éste el motivo de hospitalización más frecuente, y no se presentaron brotes. Esto coincide con lo que se describe en la literatura médica?.

Es interesante el alto porcentaje (64\%) de pacientes con bacteriemia sin síntomas digestivos, aunque se reconoce como puerta de entrada la digestiva, en la mayoría de los niños. Las complicaciones extra-intestinales focales más frecuentes son osteomielitis, artritis, neumonía y meningitis ${ }^{8}$.

En nuestra serie no se detectó meningitis en alguno de los cinco pacientes bajo 3 meses de edad, siendo ésta una complicación que se observa en este grupo etario ${ }^{8}$.
Considerando los pacientes que presentaron una enfermedad diseminada, llama la atención que casi la mitad de ellos no presentaban factores de riesgo para esta enfermedad. Si bien se aisló Salmonella sp en el hemocultivo de dos pacientes que cursaban una infección respiratoria, en ambos aparece un elemento de riesgo: el lactante con bronquiolitis a virus respiratorio sincicial había presentado diarrea la semana previa, y el paciente con diagnóstico de neumonía había tenido un bajo peso al nacer y presentaba una condición respiratoria previa.

Ninguno de los dos pacientes con osteoartritis séptica a Salmonella tenían antecedente alguno, vinculante ni inmunodepresión.

Una serie de 29 niños con bacteriemia por Salmonella zoonótica asistidos en un hospital universitario de Madrid durante un período de 11 años, arroja datos muy similares.

Otro elemento que debemos considerar es que la población que se asiste en nuestro centro proviene de medios socio-económicos deficitarios sin saneamiento, potabilidad del agua, e higiene inadecuadas ${ }^{17-20}$.

Otros factores de trasmisión descritos como contacto con animales de granja, mascotas, o incluso la alimentación con fórmulas, no fueron consideradas ${ }^{21}$.

Respecto a los aspectos microbiológicos, $S$. Enteritidis, la serovariedad prevalente en el mundo, seguida de $S$. Typhimurium ${ }^{1-4,6}$ fueron también los agentes más frecuentes aislados en nuestra serie. No se aisló en el período de estudio Salmonella Typhi ni otros serotipos no zoonóticos; esto no llama la atención en nuestro país dado lo poco frecuente de enfermedad por estos serotipos. En el período 
1990 a 2000 únicamente 14 cepas de Salmonella Typhi, fueron serotipificadas en el Uruguay. Los casos son muy esporádicos y la mayoría de ellos son casos importados 9 .

Desde el inicio de los años 90, han emergido cepas de Salmonella spp que son resistentes a una amplia variedad de antimicrobianos y son ahora una seria preocupación para la salud pública ${ }^{6}$.

Las cefalosporinas de tercera generación son los antimicrobianos actualmente recomendados como de primera línea en el tratamiento de los pacientes con infección diseminada por Salmonella $\mathrm{sp}^{1,2}$. Son alternativas azitromicina y ciprofloxacina que se reservan para aquellas situaciones de enfermedad grave y cepas susceptibles. Ampicilina y cotrimoxazol, al igual que cloranfenicol, han perdido, con alta frecuencia, su actividad sobre salmonelas no humanas. En nuestro país se ha mantenido una baja resistencia a ampicilina y cefalosporinas de tercera generación. Existen pautas nacionales sobre el uso racional de antimicrobianos en las gastroenteritis. No se recomienda el tratamiento antimicrobiano para la gastroenteritis en pacientes inmunocompetentes y sin factores de riesgo para enfermedad diseminada ya que prolonga el estado de portador y no mejora los síntomas.

Basados en los perfiles de susceptibilidad en nuestra serie, se pueden mantener las recomendaciones sugeridas hasta el momento ${ }^{12}$.

\section{Conclusiones}

Se debe tener en cuenta la infección por Salmonella spp en niños febriles con riesgo de enfermedad bacteriana diseminada, con o sin focalidad. Mantener la vigilancia epidemiológica de las infecciones por Salmonella spp pro- mueve un diagnóstico precoz y un tratamiento oportuno cuando sea necesario. Medidas como el uso racional de antimicrobianos y las prácticas adecuadas en la cocción y manejo de los alimentos pueden disminuir la incidencia de estas infecciones.

\section{Resumen}

Introducción: Salmonella sp puede causar infecciones asintomáticas, gastroenteritis, bacteriemia e infecciones focales como meningitis y osteomielitis. Objetivo: Describir aspectos microbiológicos y clínicos de las infecciones por Salmonella spp en niños en un hospital de referencia pediátrico Centro Hospitalario Pereira Rossell. Montevideo Uruguay. Material y Métodos: Estudio descriptivo y retrospectivo de pacientes en quienes se aislara Salmonella spp en el período 1 de enero de 2005 al 31 de diciembre de 2010. Resultados: Se aisló Salmonella spp en 46 niños menores de 15 años. Dieciocho eran menores de 2 años y 5 niños menores de tres meses. 24\% de los pacientes presentaba factores de riesgo (infección por VIH; enfermedad hemato-oncológica, desnutrición) y co-morbilidades (bajo peso al nacer y neumonía). No hubo fallecidos. Los serotipos más frecuentes fueron: Typhimurium y Enteritidis. La mayoría de las cepas eran sensibles a ampicilina y cefalosporinas de tercera generación. Discusión: La presentación clínica predominante fue diarrea con sangre, no se presentaron brotes. Basados en los perfiles de susceptibilidad antimicrobiana, se pueden mantener las recomendaciones hasta el momento sugeridas. Conclusiones: Se debe tener en cuenta la infección por Salmonella sp en niños febriles con riesgo de enfermedad bacteriana invasora, con o sin focalidad.

\section{Referencias bibliográficas}

1.- Ochoa T, Cleary T. Salmonella. En: Feigin and Cherry's Textbook of Pediatric Infectious Diseases.2009; 6th edition. Elsevier. Filadelfia: 1567-82.

2.- Zulfiqar Ahmed Bhutta. Salmonella. En Nelson Textbook of Pediatrics. 20 ed. Elsevier 2016: 1443-54.

3.- Miller I S, Hohmann E L, Pegues D A. Salmonella. En Mandell, Douglas and Bennett's Principles and Practice of Infectious Diseases. Mandell GL, Bennett JL, Dolin R, eds. Elsevier. 6 ed.: 2567-86.

4.- Salmonella infecciones. En Red Book. Enfermedades Infecciosas en Pediatría. Pickering, Baker, Kimberlin, Long. 30 ed. Editorial AAP. USA, 2015: 695-702.

5.- Pírez M C. Enfermedad diarreica de etiología infecciosa. En Pediatría urgencias y emergencias. Bello O. Sehabiague, Prego. De Leonardis. Tercera edición. Montevideo. 2009. Bibliomédica.

6.- Salmonella(non-typhoidal) http://www.who.int/ mediacentre/factsheets/fs139/es/ (Consultado el 28 de junio de 2017).

7.- $\quad$ Prado Muñoz S1, Bringué Espuny X, Solé Mir E, Hervera Coll G, López Gil A, Gomà Brufau AR. Bacteriemia en el curso de gastroenteritis a Salmonella. An Esp Pediatr 1997; 46 (2): 151-5.

8.- Díez Dorado R, Tagarro García A, Baquero-Artigao F, García-Miguela MJ, Uría González MJ, Peña Garcíab P, et al. Bacteriemia por Salmonella no typhi en niños: revisión de 11 años An Pediatr (Barc) 2004; 60 (4): 344-8.

9.- Acuña A, Algorta G, Alfonso A, Anchieri D, Betancor L, Chabalgoity J A, et al. Salmonella en: Enfermedades transmitidas por alimentos en Uruguay. Montevideo: OPS; 2002. OPS. Pag 16-34. http://pesquisa.bvsalud.org/cvsp/ resource/pt/lil-337141

10.- Bronquiolitis. Atención Pediátrica. Normas Nacionales de Diagnóstico, Tratamiento y Prevención. Octava edición. Montevideo. Oficina del Libro. 2014. 323-33.

11.- Infección osteoarticular. Atención Pediátrica. Normas Nacionales de Diagnóstico, Tratamiento y Prevención. Octava edición. Montevideo. Oficina del Libro. 2014; 549-59.

12.- Gastroenteritis. Atención Pediátrica. Normas Nacionales de Diagnóstico, Tratamiento y Prevención. Octava edición. Montevideo. Oficina del Libro. 2014; 217-42.

13.- Fiebre sin foco aparente. Atención Pediátrica. Normas Nacionales de Diagnóstico, Tratamiento y Prevención. Octava edición. Montevideo. Oficina del Libro. 2014; 511-22. 
14.- Neumonía. Atención Pediátrica. Normas Nacionales de Diagnóstico, Tratamiento y Prevención. Octava edición. Montevideo. Oficina del Libro. 2014. 249-364.

15.- Bello O, Sehabiague G, Prego J, de Leonardis D. Sepsis y shock séptico comunitario. En Pediatría urgencias y emergencias. Tercera edición. Montevideo. 2009. Bibliomédica 381-98.

16.- Bello O, Sehabiague G, Prego J, de Leonardis
D. Fiebre sin foco. En Pediatría urgencias y emergencias. Tercera edición. Montevideo. 2009. Bibliomédica 347-60.

17.- Uribe C, Suárez M C. Salmonelosis no tifoidea y su transmisión a través de alimentos de origen aviar. Colomb Med 2006; 37: 151.

18.- Jones T F, Ingram L A, Fullerton K E, Marcus R, Anderson B J, McCarthy P V, et al. A casecontrol study of the epidemiology of sporadic Salmonella infection in infants. Pediatrics 2006;
118 (6): 2380-87.

19.- Christenson J C. Salmonella infections Pediatric Rev 2013; 34 (9): 375-83.

20.- Chai S J, White P L, Lathrop S L, Solghan S M, Medus C, McGlinchey B M, et al. Salmonella enterica serotype Enteritidis: increasing incidence of domestically acquired infections. Clin Infect Dis 2012: 54 (Suppl 5).

21.- Hohmann E. Nontyphoidal salmonellosis. Clin Infect Dis (2001) 32 (2): 263-9. 